\title{
3D Spectroscopy of the Blue Compact Dwarf Galaxies IZw18 and IIZw70
}

\author{
C. Kehrig ${ }^{1,2}$, J. M. Vílchez ${ }^{1}$, S. F. Sanchez ${ }^{3}$, L. Lopez-Martin ${ }^{4}$, \\ E. Telles ${ }^{2}$ and D. Martin-Gordón ${ }^{1}$ \\ ${ }^{1}$ Instituto de Astrofísica de Andalucía (CSIC) \\ Camino Bajo de Huetor, Apartado 3004, 18080 Granada, Spain \\ email: kehrig@iaa.es, jvm@iaa.es \\ ${ }^{2}$ Observatório Nacional, Rua José Cristino, 77, E-20.921-400, Rio de Janeiro, Brazil \\ ${ }^{3}$ Calar Alto Observatory (CAHA), Almería E-040004, Spain \\ ${ }^{4}$ Instituto de Astrofísica de Canarias, c/ Vía Lctea s/n, 38200, La Laguna, Tenerife, Spain
}

\begin{abstract}
Blue compact dwarf (BCD) galaxies are low-metallicity objects undergoing violent star formation (Searle \& Sargent 1972). We present our ongoing work on integral field spectroscopy (IFS) of the two prototypical BCD galaxies IIZw70 and IZw18 (Papaderos et al. 2002). Two-dimensional spectroscopy allows us to collect simultaneously the spectra of many different regions of an extended object, combining photometry and spectroscopy in the same data set. The great advantage of using IFS for the investigation of galaxies is that it allows us to obtain data on the galaxies' positions, velocity fields and star-forming properties all in one data cube. The observations were taken with the instruments INTEGRAL/WYFFOS at the WHT (ORM, La Palma) and PMAS installed on the $3.5 \mathrm{~m}$ telescope at the CAHA, covering a spectral range from $\lambda 3600$ to $6800 \AA$. The data are mainly used to study the ionized gas and stellar clusters. Our main goal is to investigate the presence of spatial variations in ionization structure indicators, physical conditions and gaseous metal abundances, in these galaxies (Vílchez \& Iglesias-Páramo 1998). We also study the kinematical properties of the ionized gas, as well as systematic variations of ionization structure and physical-chemical parameters as a function of the surface-brightness in $\mathrm{H} \alpha$ emission. Maps of the relevant emission lines are presented.
\end{abstract}

Keywords. ISM: ionization structure, ISM: HII regions, Galaxies: abundances, Galaxies: dwarf
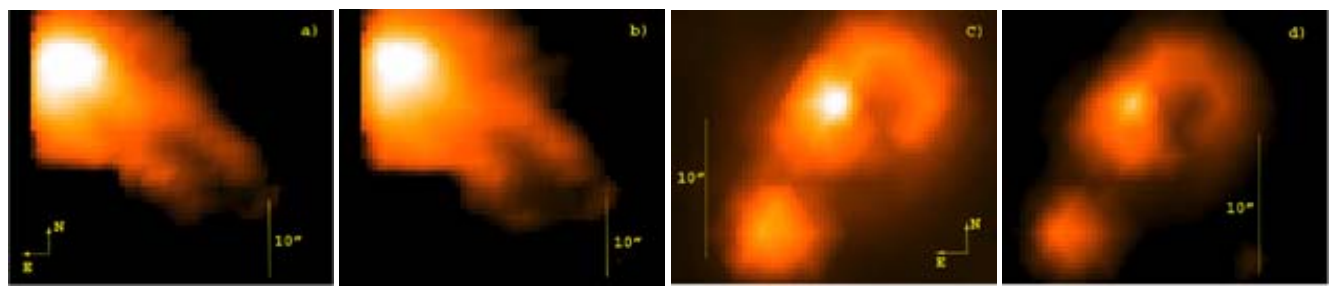

Figure 1. Maps in emission lines $\mathrm{H} \alpha \lambda 6563 \AA$ and $[\mathrm{O}$ III] $\lambda 5007 \AA$ for IIZw70 (a,b) and IZw18 $(\mathrm{c}, \mathrm{d})$, respectively. The emission line images have subtracted the continuum using images using adjacent wavelengths obtained with PMAS at CAHA and INTEGRAL/WYFFOS at WHT. North is up and east is left.

\section{References}

Papaderos, P., Izotov, Y.I., Thuan, T.X., et al. 2002, A $\& A$ A 393, 461

Searle, L. \& Sargent, W.L.W. 1972, ApJ 173, 25

Vílchez, J.M. \& Iglesias-Páramo, J. 1998, ApJ 508, 248 